\title{
Publisher Correction to: Safety, effectiveness and mid-term follow-up in 136 consecutive patients with moderate to severely calcified lesions undergoing phoenix atherectomy
}

\author{
Sorin Giusca ${ }^{1}$ Michael Lichtenberg ${ }^{2} \cdot$ Melanie Schueler $^{3}$. Ulrike Heinrich ${ }^{4} \cdot$ Christoph Eisenbach $^{5}$. \\ Martin Andrassy ${ }^{6}$. Grigorios Korosoglou ${ }^{1}$ (1)
}

Published online: 20 October 2021

(c) Springer Japan KK, part of Springer Nature 2021

\section{Publisher Correction to: \\ Heart and Vessels (2021) 36:366-375 \\ https://doi.org/10.1007/s00380-020-01695-w}

In the original publication of the article, "Appendix methods" was missing and has now been uploaded.

The original article was updated.

Electronic supplementary material The online version of this article (https://doi.org/10.1007/s00380-021-01967-z) contains supplementary material, which is available to authorized users.
Publisher's Note Springer Nature remains neutral with regard to jurisdictional claims in published maps and institutional affiliations.

The original article can be found online at https://doi.org/10.1007/ s00380-020-01695-w.

Grigorios Korosoglou

gkorosoglou@hotmail.com; grigorios.korosoglou@grn.de

1 Cardiology and Vascular Medicine, GRN Hospital Weinheim, Weinheim, Germany

2 Vascular Center Klinikum Arnsberg, Arnsberg, Germany

3 Practice for Cardiology and Vascular Medicine, Hirschberg, Germany

4 Practice for Vascular Medicine and Gastroenterology, Weinheim, Germany

5 Diabetology and Gastroenterology, GRN Hospital Weinheim, Weinheim, Germany

6 Fuerst-Stirum Hospital, Cardiology and Vascular Medicine, Bruchsal, Germany 